\title{
Recolección y manejo de recursos forestales nativos, en especial del algarrobo (Prosopis) en la región de Fiambalá, Catamarca (Argentina)
}

\section{Collection and handling of native forest resources, especially from algarrobo (Prosopis sp.) in the region of Fiambalá, Catamarca (Argentina)}

\author{
Diego Andreoni ${ }^{1,2}$, Bernarda Marconetto ${ }^{3}$, Verónica A. Mors ${ }^{4}$ y Norma Ratto 5
}

\begin{abstract}
Resumen
Se evalúa el proceso de selección y utilización de recursos leñosos en la región de Fiambalá (Departamento Tinogasta, Catamarca), tanto por las poblaciones que habitaron la región a lo largo del primer milenio de esta era como de la incaica. Se realizó el análisis antracológico de carbones procedentes de sitios arqueológicos en distintos contextos (residenciales y productivos), los cuales están emplazados en diferentes sectores y altitudes de la región (1500 a $1900 \mathrm{msnm}$ ). Los resultados dan cuenta de un uso intensivo del algarrobo (Prosopis sp.) en combinación con otras especies a lo largo del lapso considerado. Además, relacionamos y contextualizamos los resultados obtenidos con estudios previos realizados en el marco del Proyecto ArqueológicoChaschuil Abaucán y en otras áreas del Noroeste Argentino. Discutimos el uso del algarrobo como especie cultivada, proceso que se consolida en la etapa incaica.
\end{abstract}

Palabras claves: madera, Prosopis, Formativo, Tardío-Inca, Argentina.

\begin{abstract}
Management process, selection and use of woody resources in the region of Fiambalá (Department Tinogasta, Catamarca) is evaluated, both by the populations who inhabited the region throughout the first millennium of the era as the Inca. The anthracological analysis of charcoals from archaeological sites in different contexts (residential and productive), which are located in different sectors and altitudes in the region (1500-1900 masl) was performed. The results show intensive use of algarrobo (Prosopis sp.) in combination with others species throughout the period considered. Besides, we relate and contextualize the results with previous studies in the framework of Archaeological Project Chaschuil-Abaucán and other areas of the Argentine Northwest. We discuss the use of algarrobo as cultivated species, a process that is consolidated in the Inca period.
\end{abstract}

Keywords: Wood, Prosopis sp., Formative, Late-Inca, Argentina.

Recibido: 16 marzo 2016. Aceptado: 10 diciembre 2016

1 CONICET- División Arqueología, Museo de La Plata, Universidad Nacional de La Plata, Paseo del Bosque s/nº (1900) La Plata, ARGENTINA. Email: andreondieg@hotmail.com

2 CONICET (Consejo Nacional de Investigaciones Científicas y Técnicas) Argentina

3 Instituto de Antropología de Córdoba - CONICET Universidad Nacional de Córdoba, Av. H. Yrigoyen 174 (5000) Córdoba, ARGENTINA. Email: bernarda.marconetto@gmail.com

4 Instituto de Antropología de Córdoba Universidad Nacional de Córdoba, Av. H. Yrigoyen 174 (5000) Córdoba, ARGENTINA. Email: veromors@yahoo.com.ar

5 Universidad de Buenos Aires, Instituto de las Culturas (UBA-CONICET), Facultad de Filosofía y Letras, Moreno 350, CP 1091 C.A.B.A., ARGENTINA, nratto@filo.uba.ar 


\section{Introducción}

Desde sus comienzos, a mediados de la década de 1990, el Proyecto Arqueológico Chaschuil-Abaucán (en adelante PACh-A) encaró el desafío de comprender la diversidad cultural y ambiental del pasado del oeste catamarqueño a través de la adopción de una escala espacial y temporal amplia y el desarrollo de distintas líneas de investigación (producción, distribución y consumo de objetos cerámicos y líticos, lenguajes visuales, organización del espacio, funebria, consumo de recursos animales y vegetales, producción de alimentos, memoria social y estudios paleoambientales, entre otros), las cuales se articularon con otros saberes aportados por las comunidades locales (Ratto, 2013, 2015). En este largo proceso, las sociedades del primer milenio tuvieron un papel central y protagónico por constituir los grupos locales cuyos modos de vida se produjeron y reprodujeron entre los siglos I y XIII, tanto en las tierras bajas como en las altas de la región (Ratto et al., 2014). Esa continuidad se materializó en la repetición de prácticas que se transmitieron, reprodujeron, resignificaron y/o se perdieron a lo largo del tiempo y que lograron ser definidas a través del estudio de objetos, restos y lugares para conocer cuáles continuaron y cuáles cambiaron a lo largo del desarrollo temporal del extenso proceso cultural. Sostenemos que estas regularidades perduraron en el tiempo más allá del lapso fijado por la periodificación cultural tradicional del Noroeste Argentino (en adelante NOA) catamarqueño (ca. 500 AC-900/1000 DC) $y$ fueron en parte favorecidas por la ocurrencia de eventos naturales que generaron inestabilidad ambiental en el fondo de valle (Ratto et al., 2013). Esto provocó su abandono y desocupación por cientos de años hasta su recomposición y repoblamiento por la conquista incaica, con la cual ingresan a la región nuevas prácticas, tanto propias del incario como de las poblaciones movilizadas en el marco de su estrategia de dominación (Orgaz y Ratto, 2015; Ratto, 2013; Ratto y Boixadós, 2012).

En este contexto, los estudios arqueobotánicos tuvieron un rol importante dentro de las distintas líneas de evidencia implementadas por el PACh-A para configurar el proceso sociohistórico regional, los cuales fueron realizados aplicando una perspectiva etnobotánica (Pérez y Ratto, 2004) y arqueo- botánica, tanto de macrorrestos vegetales (Ratto et al., 2010, 2014) como de microrrestos (almidones) y ácidos grasos (Lantos et al., 2015). No obstante, hasta el momento no se había indagado de forma sistemática el proceso de selección y utilización de recursos leñosos en la región. En esta dirección, en el presente trabajo nos proponemos establecer qué criterios intervinieron en la selección y utilización de los recursos leńosos a partir del análisis antracológico de distintos contextos, productivos y residenciales, recuperados en sitios arqueológicos emplazados en diferentes cotas altitudinales del valle de Fiambalá, los cuales dan cuenta de un amplio lapso que se extiende desde el Período Formativo hasta el Inca. Nuestros objetivos particulares son conocer: a) si las continuidades en las prácticas sociales que evidencia el registro arqueológico para momentos de las sociedades del primer milenio (Formativo) también están reflejadas en el registro antracológico; b) los cambios y/o continuidades en el manejo de los recursos leñosos producidos por la ocupación incaica; c) el impacto que tuvo en las estrategias de selección y utilización de los recursos leñosos dicha conquista, y d) cuál fue el rol del algarrobo (Prosopis sp.) en los distintos contextos (domésticos y productivos) y en las diferentes ocupaciones del valle.

En el presente trabajo entendemos que las estrategias de selección y utilización de los recursos combustibles están enmarcadas en los patrones generales de abastecimiento de otros bienes (p.e., líticos, faunísticos, arcillas para producción cerámica, entre otros). El proceso de selección de los recursos leñosos consiste en discriminar entre una variedad de plantas cuáles son las más adecuadas para realizar diferentes tareas. Entendemos que la sola presencia de una planta leñosa en un ambiente no es criterio suficiente para que ésta sea considerada como leña, lo que define su recolección es su futura aplicación. Esta aplicación está condicionada por diversas cuestiones: las propiedades de la madera (p.e., químicas, físicas, mecánicas, organolépticas, dimensiones, entre otras); los múltiples usos que de una planta pueden presentar (p.e., medicinales, alimenticios, materia prima para producir tecnología, vestimenta, construcciones, entre otros); su valor simbólico y las necesidades de reproducción social del grupo (Andreoni, 2014; Ciampagna, 2014; Marconetto, 2008; Pique í Huerta, 1999). Habitualmente se 
supone que la recolección de madera para leña se orienta principalmente a la selección de madera muerta, no obstante, los ciclos de producción natural de madera muerta pueden ser superados por los ritmos de recolección de leña según la duración y dimensiones de las ocupaciones, la frecuencia y la intensidad de las combustiones, entre otros factores naturales y antrópicos (Andreoni, 2014; Marconetto, 2008; Pique í Huerta, 1999). En este sentido, en determinados contextos sociales las estrategias de selección de madera son diversas; pudiendo por medio de distintas técnicas silvícolas incrementar la disponibilidad de recursos maderables e incluso la producción de frutos silvestres obtenidos de un mismo taxón (p.e., poda y almacenamiento, tala sistemática, tolerancia o protección de ejemplares silvestres, erradicación de competidores, implantación bajo condiciones de cultivo, entre otros).

La región de Fiambalá está delimitada al este por las sierras homónimas, al oeste por las Sierras de Narváez, al norte por la cordillera de San Buenaventura y al sur, arbitrariamente, por el río El Puesto (Figura 1). El sector bajo es atravesado por el río Fiambalá o Abaucán que nace al norte del pueblo de Medanito (Catamarca) y desagua en la provincia de La Rioja; por lo cual constituye una de las cuencas hídricas más grandes de Argentina $\left(28.200 \mathrm{~km}^{2}\right)$, ya que recibe numerosos afluentes a lo largo de su recorrido. Esta región presentó en el pasado una dinámica particular producto de la acción volcánica y fluvial, que actuó configurando distintos escenarios paleoambientales. En este sentido, hacia los 2000 años $\mathrm{AC}$ comienza un período de transición fluvial donde se pasa de una dinámica de acumulación a la actual de encajonamiento de los cauces de ríos y erosión. El consecuente descenso del nivel de base de los cursos de agua debió haber impactado de forma negativa en las poblaciones agropastoriles del norte del valle de Fiambalá. De igual modo, la dinámica fluvial del río La Troya pudo haber afectado a las poblaciones asentadas en el sector meridional del valle (Ratto et al., 2013). Estos procesos influyeron tanto en las poblaciones del primer milenio como en los incas y españoles. Adicionalmente, el valle tuvo una importante actividad volcánica que arrojó una gran masa de material no consolidado a la superficie terrestre, lo que modificó la topografía de la región. No obstante, es probable que estos eventos catastró- ficos no sucedieran ni en tiempo de las poblaciones del primer milenio, ni durante el Estado inca. Estos procesos se retroalimentaron con otros como la acción eólica y la dinámica fluvial, que afectaron en diferente grado a las poblaciones del valle. El impacto de estos procesos sobre la vegetación es actualmente difícil de establecer. Es probable que en momentos de las poblaciones formativas y el Período Inca las condiciones fueran distintas a las actuales, no en términos fitogeograficos, sino más bien en cuanto a las frecuencias de los taxones presentes en cada ambiente. Lo más llamativo es el aparente hiatus ocupacional que se registra entre 1000 y $1250 \mathrm{DC}$, momento para el que no se han registrado asentamientos en el fondo del valle (Ratto et al., 2013).

La fitogeografía actual de la región de Fiambalá fue incluida en el dominio chaqueño, Provincia de Monte (Cabrera, 1976; Morello, 1958). En líneas generales la vegetación corresponde a un monte xerófito, diferenciándose distintas unidades. La primera de ellas son los bosques de algarrobo (Prosopis flexuosa) que crecen siempre vinculados a los cursos de agua y a napas freáticas (plantas freatofitas). En el bolsón de Fiambalá éstos se desarrollan en las proximidades del río homónimo y se extienden por el río Chaschuil hasta los $2000 \mathrm{msnm}$, aproximadamente. Otros recursos vegetales asociados a los algarrobales son chañares (Geoffroea decorticans), atamisque (Atamisquea emarginata) y quillay (Bredemeyera colletioides), entre otros (Czajka y Vervoorst, 1956; Vervoorst, 1951). Fuera del dominio de los bosques de algarrobo se extiende la estepa arbustiva conformada principalmente por jarillas (Larrea cuneifolia, $L$. divaricata y en menor proporción $L$. nitida), acompañadas de otros arbustos como brea (Cercidium praecox), usillo (Tricomaria usillo), retama (Bulnesia retama), ala de loro (Monttea aphylla), monte negro (Bougainvillea spinosa), tala de burro (Grabowskia duplicata) y huañil (Proustia ilicifolia). El estrato herbáceo está compuesto por Allionia incarnata, Junellia longidentata, Verbena critbmifolia, entre otros (Czajka y Vervoorst, 1956; Vervoorst, 1951). Esta estepa arbustiva se extiende tanto hacia el este como hacia el oeste de los bosques de algarrobo, aproximadamente hasta los $3000 \mathrm{msnm}$.

En el pasado los algarrobales debieron presentar una mayor extensión que la actual, como lo demuestran 
estudios históricos que dan cuenta de la intensa sobreexplotación a la que fueron sometidos los bosques a causa de la industria minera y ferroviaria desde fines del siglo XIX y comienzos del XX (Rojas, 2013). En el valle de Fiambalá se pueden diferenciar los algarrobales silvestres, previamente mencionados, y un segundo bosque ubicado en la localidad de El Puesto-Anillaco (Salinas, 2006 Ms.). Según Palacios y Brizuela (2005), estos últimos fueron implantados por los pobladores precolombinos, presentando una llamativa afinidad entre individuos de localidades distantes. En condiciones similares de implantación se encontrarían otros algarrobales del NOA, como por ejemplo, en Belén (Catamarca), Quilmes (Tucumán) y Tolombón (Salta). Palacios y Brizuela (2005) sostienen que la ubicación y ordenamiento de estos árboles en esos lugares solo se daría si fueron cultivados, por lo que se los puede considerar como patrimonio genético-cultural. Una de las características de este bosque es la presencia de diferentes especies de algarrobos Prosopis flexuosa, P. chilensis y una forma intermedia entre ambos (híbridos). Éstos se distribuyen de distintos modos, pudiendo encontrarse ejemplares maduros aislados en parcelas limpias, o forman cortinas rompe vientos que delimitan las parcelas despejadas o distribuidos uniformemente a manera de cultivo forestal (Salinas, 2006 Ms.). La sistemática de Prosopis sp. fue establecida por Burkart (1976), quien tras realizar una extensa revisión del género, divide las especies existentes en cinco secciones. En cada sección los taxones que la integran son similares morfológicamente y se hibridizan con facilidad, esto último se debe a que los algarrobos son genéticamente poliploides, confiriendo una gran plasticidad fenotípica que les permite adaptarse fácilmente a distintas condiciones ambientales (Beresford-Jones, 2011; Pasiecznik et al., 2001). Entre éstas, la sección definida por Burkart (1976) como Algarrobia es la de mayor importancia para el presente trabajo. La misma incluye 35 especies nativas de Sudamérica de las 45 existentes. Esto llevó a Burkart y Simpson (1977) a proponer que el género se originó en el continente sudamericano y que las diferentes especies responden a episodios de aislamiento que han producido una especiación parcial, inferencias que posteriormente fueron confirmadas por estudios moleculares (Beresford-Jones, 2011; Pasiecznik et al., 2001). Ahora bien, en términos adaptativos los alga- rrobos presentan distintas características anatómicas y morfológicas que les permiten sobrevivir en condiciones de extrema aridez, entre las que podemos mencionar el desarrollo de extensos sistemas radiculares, la presencia de hojas pequeñas que disminuyen la pérdida de agua por evaporación a la vez que facilitan la condensación de humedad durante las noches, entre otras (Beresford-Jones, 2011). Una de las características principales de los algarrobos, y por la cual son valorados por las poblaciones humanas, es que pueden ser consideradas plantas multipropósito, dado que permiten obtener distintos bienes de consumo. Entre éstos, sus frutos son comestibles, su madera dura o semidura es un excelente combustible, además de ser utilizada para la construcción y sus hojas y frutos poseen propiedades medicinales. Se suma a esto que, en términos ecológicos, son fijadores de nitrógeno en suelos y evitan su erosión (BeresfordJones, 2011; McRostie, 2014; Pasiecznik et al., 2001, entre otros). Algunas de estas características han sido consideradas para explicar la existencia de Prosopis alba en Atacama. En este sentido McRostie (2014, 2016), tras un minucioso relevamiento del registro paleoambiental y arqueológico de macrorrestos y microrrestos, observa la ausencia de registro vinculada a este taxón durante el Arcaico y su paulatino incremento a nivel de microrrestos y macrorrestos en el Período Formativo de Atacama (McRostie, 2014; 2016). En esta misma región ya se había propuesto la importancia del algarrobo y del chañar para los grupos del Arcaico y para las sociedades Formativas (Agüero y Uribe, 2011; Vidal, 2006). En la costa sur de Perú se ha propuesto una situación similar, para explicar los relictos de bosque de huarangos (complejo Prosopis juliflora-P. pallida ${ }^{6}$ ) los cuales permitieron el desarrollo de sociedades agropastoriles en contextos desérticos durante el Horizonte Medio de Nazca (ca. 1000 años DC) (Beresford-Jones, 2011; Beresford-Jones et al., 2011).

6 El complejo Prosopis juliflora-P. pallida fue definido por Pasiecznik y colaboradores (2001). Se trata de dos especies que tienen su origen en zonas tropicales de Centroamérica y/o Perú y poseen una gran afinidad genética y tolerancias similares (no resisten las heladas); además de la morfología de sus frutos, hojas, flores y en la estructura del árbol, por lo cual el autor las considera complejo Prosopis juliflora-P. pallida, definición que es aplicada por Beresford-Jones (2011) a los algarrobos de Perú. 


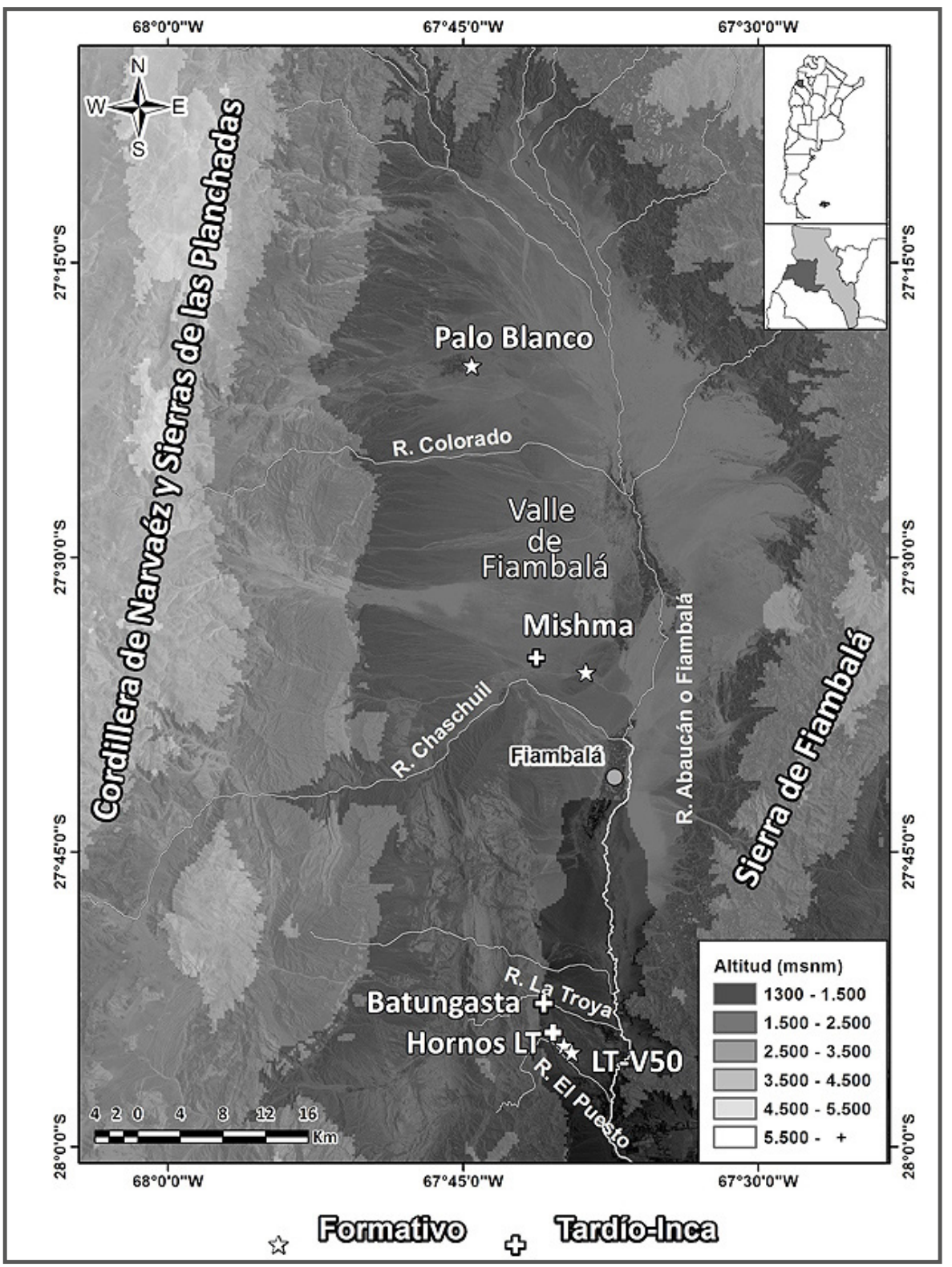

Figura 1. Sitios y localidades arqueológicas de proveniencia de las muestras antracológicas y su ubicación temporal relativa.

\section{Sitios arqueológicos y materiales}

\section{Sector norte de la región de Fiambalá}

La localidad arqueológica de Palo Blanco es la única aldea formativa conocida y emplazada en el sector norte del valle de Fiambalá, a 1900 msnm, donde en la actualidad domina una cubierta vegetal formada por arbustos de monte bajo con presencia de jarilla
(Larrea sp.), brea (Cercidium praecox) y retama (Bulnesia retama), entre otras.

Los trabajos de investigación fueron iniciados en la década de 1960 (Sempé, 1976 Ms.), cuando se registraron cinco núcleos habitaciones $(\mathrm{NH}-01 \mathrm{a}$ 05) construidos con muros de tapia y con diferentes arreglos arquitectónicos. Éstos están compuestos por tres o cuatro recintos, predominantemente 
rectangulares, que se conectan entre sí y con el exterior a través de pasillos más estrechos; en algunos casos están asociados con espacios más amplios que fueron interpretados como patios. Posteriormente, los trabajos fueron retomados por el Proyecto Arqueológico Chaschuil-Abaucán durante la década del 2000, cuando se realizaron intervenciones en el $\mathrm{NH}-03$ y en un nuevo núcleo denominado $\mathrm{NH}$ 06 con basural externo, los que fueron identificados mediante técnicas y métodos geofísicos (Bonomo et al., 2010; Martino et al., 2006), los que posteriormente fueron excavados (Ratto, 2007; Ratto y Basile, 2010).

Recientemente se han realizado excavaciones al sur

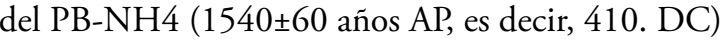
donde Sempé (1976 Ms.) identificó un basural y cuya visibilidad actual resulta nula. El sondeo se practicó en un área rodeada de excavaciones asistemáticas realizadas por pobladores locales, evidenciadas por depresiones en la superficie del terreno y por distintos tipos de materiales arqueológicos asociados (cerámica, lítico y óseo). El sondeo realizado no presenta en su estratigrafía evidencia de algún tipo de alteración reciente, por lo que se asume que los materiales recuperados son el resultado de procesos de depositación en los que no intervinieron las excavaciones asistemáticas mencionadas anteriormente. Se alcanzó una profundidad máxima de $1.95 \mathrm{~m}$ respecto del nivel actual del terreno y se identificaron seis capas estratigráficas naturales de distintos espesores y características sedimentarias. Los fechados radiométricos indican concordancia en función de las profundidades de recuperación de las muestras (Tabla 1). Por lo expuesto, las muestras antracológicas analizadas provienen de $\mathrm{NH}-03$ y NH-06 y de los basureros ubicados al este del NH-06 (Miyano et al., 2015) y al sur del NH-04, los cuales en conjunto cubren más de 1200 años, ya que se extienden desde 262 AC hasta 986 DC.

\section{Sector medio de la región de Fiambalá}

En este sector destaca la localidad arqueológica de Mishma, emplazada en los alrededores del Zanjón de Apocango a $1750 \mathrm{msnm}$, la que está compuesta por 10 sitios reportados por Sempé (1976 Ms.), quien realizó en algunas recolecciones de material superficial, y en otros, excavaciones mediante la técnica de trincheras. Las muestras antracológicas provienen de los sitios enumerados como 1, 2 y 7. Los dos primeros (Mishma-1 y Mishma-2) no presentan un patrón arquitectónico definido, posiblemente debido al uso de materiales perecederos en su construcción (Sempé, 1976 Ms.); sin embargo, identificó grandes fogones de donde extrajo muestras de carbón y otros restos vegetales carbonizados (abundantes semillas de chañar y algarrobo, así como marlos de maíz). En ambos sitios se recuperaron en superficie fragmentos cerámicos de características tecno-morfo-decorativas del Período Tardío y en menor medida del Temprano. En cambio, el otro sitio (Mishma-7) presenta una arquitectura definida por dos grandes núcleos arquitectónicos (IV y V) en buen estado de preservación, los cuales conforman varios recintos de muros dobles y simples, circunscritos por un muro perimetral (Orgaz et al., 2007; Sempé, 1983). Los estudios de tecnología cerámica realizados en los últimos años determinaron la presencia de un número mínimo de 35 piezas cerámicas en Mishma-7, donde el material incaico representa el $14,3 \%$ y el de filiación Tardía el $85,7 \%$ (Orgaz et al., 2007). Los fechados radiométricos con los que cuenta esta localidad informan que Mishma-2 y Mishma-7 se ubican en el siglo $\mathrm{XV}$, pero Mishma-1, a diferencia de lo sostenido por Sempé (1976 Ms.), remite a momentos formativos (ver Tabla 1 ).

Actualmente en la localidad existe una estepa de arbustiva xerófita abierta, con un suelo arenoso compacto, cubierto por una capa delgada de ripio. La vegetación es baja y rala, observándose brea (Cercidium praecox) y retama (Bulnesia retama). En prospecciones realizadas se documentaron tocones de algarrobo (Prosopis sp.) en los alrededores de Mishma-7, lo que hace pensar en la posible existencia de bosques en las inmediaciones del sitio al momento de su ocupación.

\section{Sector sur de la región de Fiambalá}

En este sector del valle se destacan el sitio incaico Batungasta, los Hornos de La Troya y la aldea La Troya-V50 (LT-V50), cuyas dataciones radiométricas dan cuenta de una ocupación del espacio, como mínimo, desde el siglo IV a momentos hispano-indígenas (Ratto, 2013) (ver Tabla 1). 
Tabla 1. Fechados radiométricos de los sitios de proveniencia de las muestras analizadas

\begin{tabular}{|c|c|c|c|c|}
\hline $\begin{array}{l}\text { Región } \\
\text { Fiambalá }\end{array}$ & Sitio & Material & $\begin{array}{l}\text { Laboratorio y fechado } \\
\text { radiométrico (AP) }\end{array}$ & $\begin{array}{l}\text { Años cal DC., } 1 \text { sigma, curva } \\
\text { SHCal13, Calib7.04 }\end{array}$ \\
\hline \multirow{8}{*}{ Norte } & \multirow[t]{2}{*}{ PB-NH6 } & $\begin{array}{l}\text { Intramuro, carbón dentro } \\
\text { de tapia }\end{array}$ & AA81736; $1194 \pm 37$ & cal DC 876: cal DC 976 \\
\hline & & Intramuro, carbón & AA81735; $1236 \pm 37$ & cal DC 832: cal DC 888 \\
\hline & $\begin{array}{l}\text { PB-NH6 } \\
\text { (basurero) }\end{array}$ & Basurero, carbón (base) & LP-2652; $1190 \pm 50$ & cal DC 861: cal DC 986 \\
\hline & \multirow{2}{*}{ PB-NH3 } & Carbón & AA65138; $1566 \pm 39$ & cal DC 517: cal DC 595 \\
\hline & & $\begin{array}{l}\text { Vegetales carbonizados } \\
\text { (cesto quemado) }\end{array}$ & AA65139; $1467 \pm 39$ & cal DC 600: cal DC 650 \\
\hline & \multirow{3}{*}{$\begin{array}{c}\text { Basurero } \\
\text { (sur PB-NH4) }\end{array}$} & Carbón, Estrato C & LP- $3187 ; 1790 \pm 70$ & cal DC 214: cal DC 376 \\
\hline & & Carbón, Estrato D & LP- $3182 ; 1940 \pm 60$ & cal DC 44: cal DC 166 \\
\hline & & Carbón, Estrato F & LP- $3184 ; 2210 \pm 80$ & cal DC 262: cal DC 137 \\
\hline \multirow{4}{*}{ Medio } & \multirow{2}{*}{ Mishma-7 } & \multirow{2}{*}{ Carbón } & AA69979 (1); $491 \pm 33$ & cal DC 1429: cal DC 1457 \\
\hline & & & AA69979 (2); $514 \pm 35$ & cal DC 1422: cal DC 1449 \\
\hline & Mishma-2 & Carbón & LP- 2647; $500 \pm 50$ & cal DC 1410: cal DC 1462 \\
\hline & Mishma-1 & Carbón & LP- $2638 ; 1470 \pm 60$ & cal DC 579: cal DC 665 \\
\hline \multirow{9}{*}{ Sur } & Batungasta* $^{*}$ & Gramínea dentro de adobe & $\mathrm{AC} 172 ; 380 \pm 60$ & cal DC 1536: cal DC 1626 \\
\hline & \multirow{4}{*}{$\begin{array}{l}\text { Hornos La Troya } \\
\text { (forma ojo de } \\
\text { cerradura o pera) }\end{array}$} & BATH-20, carbón & AA95555; $551 \pm 35$ & cal DC 1407: cal DC 1436 \\
\hline & & BATH 24/2, carbón & LP-2334; 670 40 & cal DC 1332: cal DC 1362 \\
\hline & & BATH-24/1, carbón & AA95557; $642 \pm 35$ & cal DC 1317: cal DC 1354 \\
\hline & & BATH-03, carbón & AA95556; $1096 \pm 36$ & cal DC 972: cal DC 1025 \\
\hline & \multirow{3}{*}{$\begin{array}{l}\text { Hornos La Troya } \\
\text { (forma circular) }\end{array}$} & BATH-35 (LT-V50) carbón & $\mathrm{AC}-1716 ; 1350 \pm 60$ & cal DC 656: cal DC 769 \\
\hline & & BATH-31/2, carbón & AA93280; $1305 \pm 34$ & cal DC 681: cal DC 746 \\
\hline & & BATH-36, carbón & LP- 2659; $1160 \pm 40$ & cal DC 893: cal DC 939 \\
\hline & LT-V50 & $\begin{array}{c}\text { Intramuro, } \\
\text { carbón dentro de tapia }\end{array}$ & $\mathrm{AC} 1718 ; 1250 \pm 85$ & cal DC 764: cal DC 894 \\
\hline
\end{tabular}

* En Batungasta solo se consignan los fechados de momentos incaicos, el resto son hispano-indígenas y puede consultarse en Ratto (2005).

La instalación inca de Batungasta se encuentra emplazada en la cuenca inferior del río La Troya, afluente del Fiambalá, a 1480 msnm. Presenta diversas estructuras arquitectónicas de formas rectangulares, circulares y poligonales, entre las que se hallan dos grandes plazas, recintos y cierres de recintos que definen sectores hipotéticamente abiertos (Orgaz et al., 2007; Orgaz y Ratto, 2015). Las dataciones radiométricas indican que su edificación fue en el siglo XV, pero continuó en época histórica (Ratto, 2005). Sin embargo, debido a los intensos procesos de formación, no pudo establecerse una ocupación previa a la inca dentro del área del entramado de la instalación, pero sí en su periferia sur, donde se emplazó la aldea LT-V50 de tiempos formativos (Ratto et al., 2013; ver más adelante).
Batungasta es el asentamiento de mayores dimensiones en el oeste de Tinogasta y su relevancia regional está dada por las actividades desarrolladas tanto de eventos festivos como de producción alfarera (Orgaz et al., 2007; Ratto et al., 2002). Por un lado, el comensalismo político pudo establecerse a partir del análisis de su arquitectura pública y de la evidencia artefactual cerámica. El análisis de esta última determinó la existencia de un número mínimo de 75 piezas compuestas por aríbalos, aribaloides, platos pato, ollas pie de compotera, pucos y vasijas de tamaños varios. Las piezas de filiación inca represen$\tan$ un $25 \%$, mientras que las locales alcanzan un $72 \%$ (Orgaz et al., 2007). Además, el sitio funcionó como centro de manufactura que proveyó de bienes a los sitios incaicos localizados en la alta cordillera 
andina (Ratto et al., 2002). Pero la producción de cerámica se registra desde tiempos anteriores al incario, dado que el alfar de La Troya fue explotado a lo largo del tiempo en función de la articulación de diferentes líneas de evidencia, destacándose: a) estudios de procedencia de materias primas cerámicas; b) excavaciones en estructuras de combustión (hornos) de forma circular y de ojo de cerradura o pera, localizadas en la periferia del sitio ubicadas temporalmente en los siglos VII y XV (ver Tabla 1), los cuales son denominados Hornos de La Troya; c) alta frecuencia de fragmentos cerámicos defectuosos; d) existencia de un bosque relictual de algarrobo (Prosopis flexuosa y P. chilensis) que fuera implantado por las poblaciones prehispánicas (Palacios y Brizuela 2005), y e) abundante disponibilidad de bancos de arcilla en el área de La Troya (Feely, 2011; Ratto et al., 2002, 2004, entre otros).

En los Hornos La Troya, contexto productivo, se registraron 60 estructuras de combustión, aisladas y/o asociadas, las que presentan tres tipos de formas: a) circular; b) pera u ojo de cerradura, y c) subcuadrangular (Feely 2011). Se analizaron muestras provenientes de los hornos circulares (BATH-31/2, BATH-35 y BATH-36) y forma de pera u ojo de cerradura (BATH-24/2 y BATH-03), siendo los primeros más tempranos que los segundos (ver Tabla 1). Se aclara que otros hornos fueron intervenidos, pero el carbón recuperado solo fue suficiente para realizar un fechado radiométrico.

Por último, la aldea LT-V50 se emplaza en el margen derecho de un brazo inactivo del río La Troya, a unos $4 \mathrm{~km}$ al sur del sitio Batungasta. La instalación está localizada dentro de un ambiente de barreal bisecado por antiguos cursos de agua y, en algunos sectores invadidos por sedimentos eólicos que forman médanos en pequeña escala. En terreno se visualizan segmentos de muros de tapia sin continuidad espacial ni definición certera de las formas de los recintos debido al mal estado de preservación, pero se estima que cubren una superficie de $200 \mathrm{~m}$ x $100 \mathrm{~m}$, aproximadamente. Una de las características de estos muros de tapia es que contienen pequeños carbones en su interior, hecho que también fue registrado en uno de los núcleos habitacionales (NH-06) de la localidad arqueológica de Palo Blanco (Ratto y Basile, 2010). Estos rasgos arqui- tectónicos están asociados con una alta densidad de fragmentos cerámicos en superficie, los cuales por sus características tecno-morfo-decorativas remiten a las poblaciones del primer milenio (Feely y Ratto, 2009). Además, se asocia con estructuras de forma circular, las que fueron interpretadas como hornos para la producción de cerámica (Feely, 2010; Ratto, 2005). El registro y la recuperación de material orgánico permitió obtener los fechados radiométricos que ubican temporalmente a la instalación entre 640 a 790 DC (ver Tabla 1).

\section{Metodología}

Para la identificación de los restos antracológicos se utilizó una metodología estándar (Andreoni, 2014; Marconetto, 2008; Pique í Huerta, 1999, entre otros), la cual consistió en la identificación taxonómica de los carbones arqueológicos por medio de la comparación con muestras de referencias de especies leñosas que crecen en el NOA. La unidad de análisis seleccionada fue cada uno de los carbones estudiados (Pique í Huerta, 1999), los cuales fueron fracturados a mano para el reconocimiento de los rasgos diagnósticos en corte transversal (CT), longitudinales tangencial (CLT) y radial (CLR). Los estudios se realizaron con Microscopio Óptico con luz de incidencia con un máximo de aumento de 50X. Las determinaciones fueron realizadas en dos momentos $y$ con diferentes muestras de referencia. Por una parte, se utilizó la colección depositada en el IDACOR (CONICET-Universidad Nacional de Córdoba). Por otra, fue utilizada la colección de maderas del Laboratorio 129 de la División de Arqueología Museo de La Plata, que cuenta con especies leńosas que abarcan la Diagonal Árida Argentina. La cantidad de carbones analizados en cada sitio y contexto fue variada, ya que en aquellos casos donde la muestra era reducida se analizó el total; mientras que en los contextos con gran cantidad de carbones se obtuvo una submuestra definida a través de la utilización de la curva de riqueza específica (Andreoni, 2014; Marconetto, 2008; Pique í Huerta, 1999).

Los datos se presentan y sintetizan utilizando distintas tablas y gráficos, donde en ambos casos se considera tanto la frecuencia absoluta de carbones (cantidad de carbones de cada taxón) como el volumen expresado en milílitros. Se realizaron dos agru- 
pamientos: a) el primero consideró la procedencia geográfica de las muestras (sectores norte, centro y sur de la región de Fiambalá); mientras que; b) el segundo, se realizó sobre la base de las dataciones disponibles que permiten definir dos bloques temporales: el que remite al desarrollo de las sociedades formativas y el que dirige a las sociedades tardíoincaicas que se asentaron en la región después del 1300 DC, aproximadamente.

\section{Resultados}

En total se analizaron 324 carbones de los cuales se logró identificar el 98,5\%. Se reconocieron 10 taxones, los cuales representan distintos porcentajes de la muestra total: Prosopis sp. (60\%), Geoffroea decorticans (11,7\%), Bulnesia aff. retama (10,8\%), Larrea sp. (4\%), Lithraea aff. molleoides (3,7\%), Acacia caven/Prosopis ferox (3,1\%), Schinus sp. $(1,8 \%)$, Rhamnaceae (1,5\%), Acacia sp. (1,2\%), Cercidium praecox $(0,6 \%)$, e Indeterminables $(1,5 \%)$. Los resultados se presentan en la Tabla 2 y en la Figura 2. En la primera se expresan en frecuencias absolutas (cantidad de carbones) y en volumen $(\mathrm{ml})$, y en la segunda se expresan en porcentaje (\%) del volumen de cada taxón en los distintos sitios analizados, los cuales se ordenan cronológicamente. En líneas generales se pueden realizar distintas apreciaciones, basadas en la presencia/ausencia de un taxón en cada contexto. A saber:

a) Prosopis sp es el género mejor representado, identificándose en casi todos los sitios estudiados (excepto en NH-03 de Palo Blanco), durante toda la larga secuencia temporal de casi 1200 años, tanto en contextos residenciales como productivos; además, junto con Geoffroea decorticans son los únicos dos taxones presentes en los tres sectores del área de estudio.

b) Existen diferencias entre los taxones presentes en cada uno de los sectores (nortecentro-sur) de la región de Fiambalá. Se observa que Bulnesia aff. retama, Rhamnaceae y Cercidium praecox solo fueron identificados en la localidad de Palo Blanco, ubicada en el sector norte de la región; mientras que Lithraea aff. molleoides y Larrea sp. en los Hornos de la Troya y Batungasta, respectivamente, ambos localizados en el sector sur de la región.

c) La riqueza taxonómica es mayor en sitios residenciales que en los productivos. Batungasta y Palo Blanco presentan siete taxones cada uno; mientras que en los Hornos La Troya solo la mitad o menos.

Si se consideran los tres sectores estudiados se observan variaciones tanto en los términos de cantidad de fragmentos, volumen y riqueza taxonómica (ver Tabla 2). Así, en el sector norte se identificó un total de siete taxones (Prosopis sp., Bulnesia aff. retama, Acacia sp., Geoffroea decorticans, Schinus sp., Rhamnaceae, Cercidium praecox). En términos de cantidad absoluta de fragmentos y de volumen, se observa que Bulnesia aff. retama es el mejor representado, seguido de Prosopis sp. y en menor cantidad Rhamnaceae, Cercidium praecox, Geoffroea decorticans y Schinus sp. En el sector central de la región se identificó Prosopis sp. y Geoffroed decorticans, donde esta última es la especie mejor representada en términos de frecuencia absoluta de fragmentos y volumen (ver Tabla 2 y Figura 2). En el sector sur se analizaron los carbones procedentes de contextos residenciales y productivos de los sitios Batungasta, Horno La Troya y LV-50, identificándose un total de siete taxones (Acacia caven/Prosopis ferox, Geoffroea decorticans, Larrea sp., Lithraea aff. molleoides, Prosopis sp., Rhamnaceae, Schinus sp.). En términos de número de fragmentos y volumen, es Prosopis sp. el género mejor representado seguido por Geoffroea decorticans, Larrea sp., Lithraea aff. molleoides, Acacia caven/Prosopis ferox; finalmente en menor proporción se identificó Rhamnaceae y Schinus sp.

Al comparar los resultados obtenidos, entre los contextos analizados observamos algunas diferencias, en primer lugar, la más evidente es la ya mencionada en términos de riqueza taxonómica. En segundo lugar, la identificación de distintos taxones en cada contexto, por ejemplo, Lithraea aff. molleoides está presente únicamente en los hornos, mientras que Larrea sp., Rhamnaceae, Schinus sp., solo en contextos domésticos. En los dos tipos de formas de los Hornos de La Troya (circular y forma de pera u ojo de cerradura) se identificó Prosopis sp. Entre los circulares BATH-36 y BATH-35 fue el único género 
Tabla 2. Resultado general de taxones identificados en la región de Fiambalá

\begin{tabular}{|c|c|c|c|c|c|c|c|c|c|c|c|c|c|c|c|c|c|c|c|c|c|c|c|c|}
\hline : & 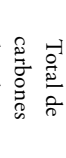 & $\frac{\bar{z}}{\tilde{\sigma}}$ & & 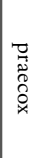 & & 莡 & & 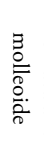 & & 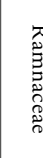 & & 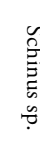 & & 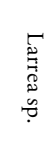 & & 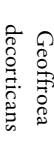 & & 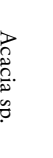 & 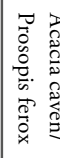 & & 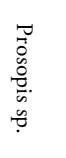 & \multirow[t]{2}{*}{ 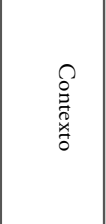 } & & \\
\hline$\lesssim$ & $z_{0}$ & $\lesssim$ & $z_{0}$ & $\lesssim$ & $z_{0}$ & $\lesssim$ & $z_{0}$ & $\lesssim$ & $z_{0}$ & $\lesssim$ & z & $\lesssim$ & $z_{0}$ & $\lesssim$ & $z_{0}$ & 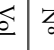 & $\lesssim$ & $z_{0}$ & 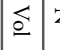 & \begin{tabular}{l|l}
$z_{0}$ & $\oint$
\end{tabular} & $\delta z_{0}$ & & & 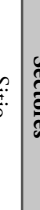 \\
\hline$\vec{\xi}$ & 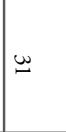 & $\vec{u}$ & - & ' & ' & $\overrightarrow{0}$ & w & ' & ' & ' & ' & ' & ' & ' & & & & ' & . & & ' & 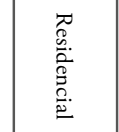 & 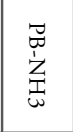 & \\
\hline$\underset{\downarrow}{\breve{~}}$ & $G$ & ' & ' & $=$ & N & ث & $u$ & ' & & $\widetilde{M}$ & - & & ' & ' & & \begin{tabular}{l|l}
$\infty$ & $N$
\end{tabular} & & ' & ' & & vu & 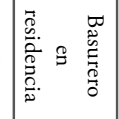 & 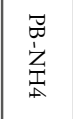 & \\
\hline$\Phi$ & $\omega$ & ' & ' & & ' & ' & & ' & & $A$ & - & & ' & ' & & ' & & . & . & & $D_{n}$ & 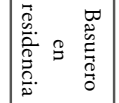 & 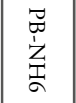 & \\
\hline I & o & ' & ' & & ' & ' & & ' & & N & - & $N$ & - & ' & & ' & $u$ & $A$ & ' & ' & A & 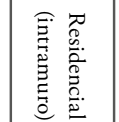 & 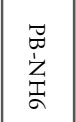 & \\
\hline$\vec{v}$ & ur & ' & ' & ' & ' & ' & & ' & ' & ' & ' & ' & ' & ' & & ' & ' & ' & ' & ' & $\vec{v} \mid u$ & 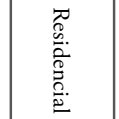 & 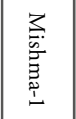 & \\
\hline$\vec{A}$ & ur & ' & ' & ' & . & ' & & ' & . & . & ' & ' & ' & ' & & A -1 & & ' & ' & ' & $5+$ & 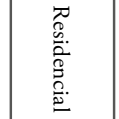 & $\begin{array}{l}3 \\
\text { 章 } \\
\text { 总 } \\
\text { in }\end{array}$ & $\stackrel{\overbrace{}}{\mathscr{E}}$ \\
\hline జّ & $\tilde{\sigma}$ & ' & ' & ' & ' & ' & ' & ' & ' & . & ' & ' & , & ' & & 8 & & ' & ' & ' & $\omega$ & 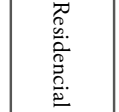 & 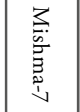 & \\
\hline$\underset{\omega}{\sim}$ & $\vec{v}$ & ' & ' & & ' & ' & . & . & . & . & ' & ' & ' & ' & & ' & & ' & . & ' & 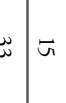 & 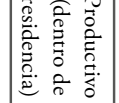 & 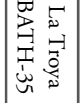 & \\
\hline ○ & 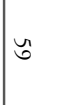 & ' & ' & ' & ' & ' & . & ' & . & ' & ' & ' & . & ' & & $N$ & 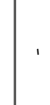 & ' & $\stackrel{N}{\sim}$ & $\overrightarrow{0} t$ & w & 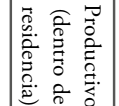 & 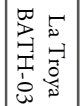 & \\
\hline w & 6 & $\vec{u}$ & - & . & ' & . & ' & $\vec{u}$ & $=$ & . & ' & ' & ' & ' & I & ' & ' & ' & ' & ' & \begin{tabular}{c|l}
0 & $v$
\end{tabular} & 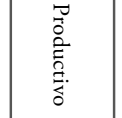 & 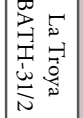 & z \\
\hline$\tilde{\sigma}$ & 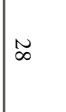 & ' & ' & & ' & . & & N & - & . & ' & . & . & ' & & ' & & ' & . & $\bar{\alpha}$ & $\vec{\infty} \mid \sim N$ & 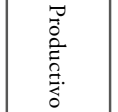 & 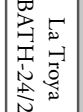 & \\
\hline$\infty$ & $\omega$ & ' & . & . & ' & ' & . & ' & ' & ' & ' & ' & ' & ' & ' & ' & . & ' & ' & ' & \begin{tabular}{c|c}
$\infty$ \\
\end{tabular} & 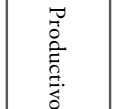 & 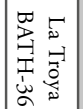 & \\
\hline$\underset{\infty}{\infty}$ & $\Xi$ & 点 & $N$ & & ' & ' & ' & ' & . & $\tilde{N}$ & $N$ & $\vec{\sigma}$ & $\backsim$ & 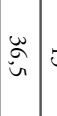 & 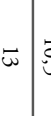 & \begin{tabular}{c|c} 
\\
$\dot{c}$
\end{tabular} & 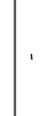 & ' & . & 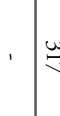 & $\vec{\omega}$ & 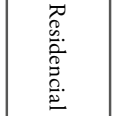 & 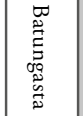 & \\
\hline వ్ర & 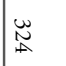 & $\ddot{\sim}$ & $A$ & $\exists$ & 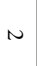 & U & w & $\underset{w}{w}$ & $\vec{N}$ & 岕 & & $\vec{N}$ & $\sigma$ & $\begin{array}{l}\text { 崩 } \\
\text { ज. }\end{array}$ & $\vec{\omega}$ & 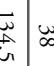 & $u$ & $A$ & $\stackrel{N}{\sim}=$ & $\left.\overrightarrow{0}\right|_{+} ^{+\infty}$ & $\vec{v}$ & & $\overrightarrow{\underline{\partial}}$ & \\
\hline
\end{tabular}




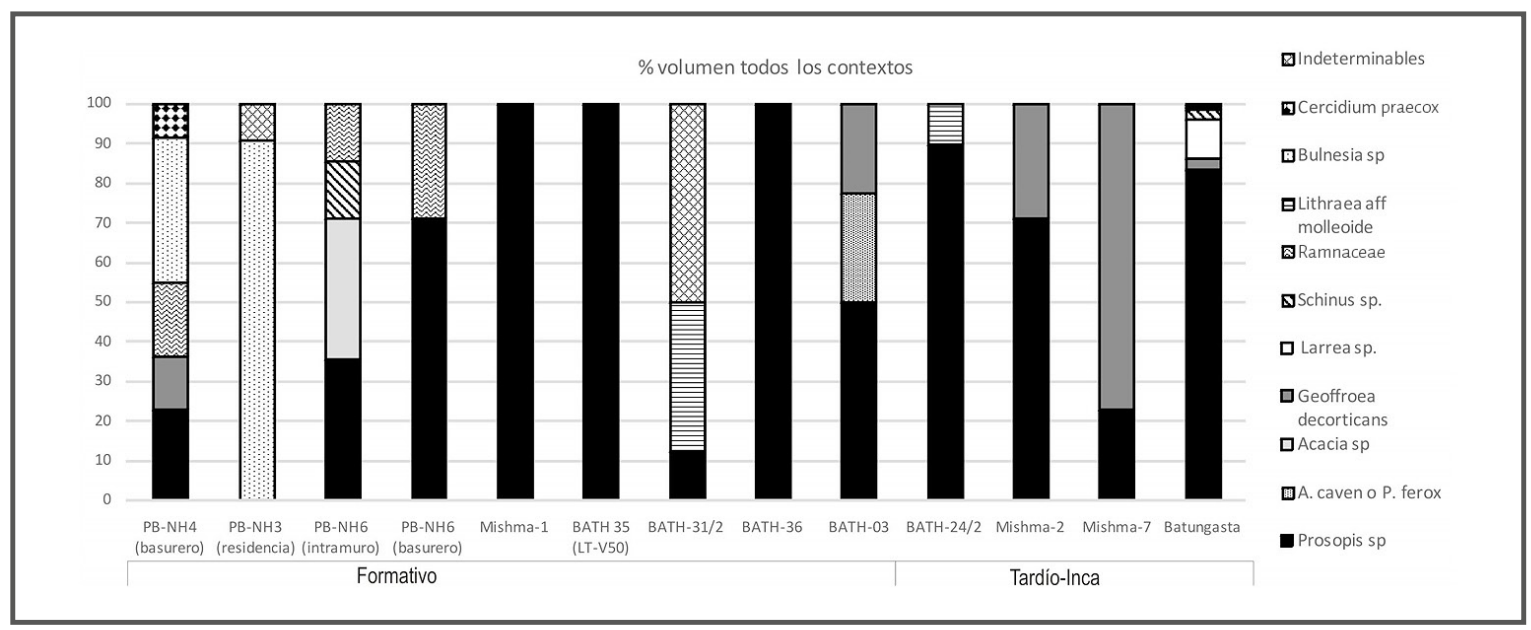

Figura 2. Porcentaje del volumen en ml de cada taxón identificado en los distintos sitios estudiados en el valle de Fiambalá.

identificado, mientras que en BATH-31/2 se asocia con Lithraea aff. molleoides. En los de forma de ojo de cerradura o pera es en BATH-03 donde Prosopis sp. se asocia a Geoffroea decorticans, Acacia caven/ Prosopis ferox, mientras que en BATH 24/2 se identificó junto con Lithraea aff. molleoides, aunque en este caso Prosopis sp. es el taxón mejor representado (ver Tabla 2, Figura 2).

Ahora bien, en términos cronológicos se observan algunas diferencias y similitudes en la utilización de recursos leñosos (ver Figura 2). En este sentido si se consideran las ocupaciones correspondientes al Período Formativo, se identificó un total de nueve taxones (Prosopis sp., Geoffroea decorticans, Acacia caven/Prosopis ferox, Lithraea aff. molleoides, Schinus sp., Acacia sp., Rhamnaceae, Cercidium praecox, Bulnesia aff. retama); mientras que para momentos de la ocupación incaica se registró una menor riqueza taxonómica (Prosopis sp., Lithraea aff. molleoides, Geoffroea decortincans, Larrea sp., Schinus sp., Rhamnaceae). Es importante resaltar la presencia de Prosopis sp. en toda la secuencia temporal dentro de los tres sectores de la región. En los Hornos de La Troya se observa una mayor riqueza en contextos productivos del primer milenio (Prosopis sp., Lithraea aff. molleoides, Geoffroea decortincans, Acacia caven/Prosopis ferox), con respecto a los más tardíos, donde solo se identificaron dos taxones (Prosopis sp. y Lithraea aff. molleoides). En cuanto a los contextos domésticos, se observan cambios o sustituciones de taxones, los cuales pueden estar vinculados a distintas actividades del grupo social o a distintas estrategias de abastecimiento de leña. En este sentido en los contextos del primer milenio se identificó Cercidium praecox, Bulnesia sp. y Acacia sp. que están ausentes en el contexto residencial incaico; mientras que Larrea sp. está presente en este último y ausente durante el primer milenio, los restantes taxones fueron identificados en ambos contextos temporales.

\section{Discusión}

Los estudios arqueobotánicos en la región de Fiambalá aportaron nuevas evidencias para comprender su dinámica poblacional. El análisis antracológico constituye el primer estudio de macrorrestos carbonizados provenientes de distintos contextos regionales (residenciales y productivos), además de cubrir una amplia cronología que se extiende desde el 266 $\mathrm{AC}$ con las sociedades formativas hasta momentos de la ocupación incaica en la región. Estos estudios específicos pueden relacionarse con otros que también fueron generados por la arqueobotánica, pero sobre otros tipos de macrorrestos, los cuales provienen de la puna transicional y el piso altoandino de la región de Chaschuil (Pérez y Ratto, 2004; Ratto et al., 2010) y la de Fiambalá (Ratto et al., 2010).

Los registros arqueobotánicos provenientes de sitios ubicados en distintas ecozonas de las regiones de 
Chaschuil y Fiambalá evidencian la complementariedad de pisos ecológicos para lograr las estrategias de subsistencia, dada la presencia de especies propias de un ambiente en sitios emplazados en otros, tanto en contextos de las sociedades del primer milenio como la tardía-incaica (Ratto et al., 2010). Ejemplos de esto lo constituyen los postes y vigas de Prosopis sp. y frutos de Geoffroea decorticans y un bastón de caminante confeccionado en Larrea sp. en el sitio multicomponente Fiambalá I (5000 msnm), dado que todas son plantas nativas de pisos ecológicos del fondo de valle. Caso contrario lo constituye la recuperación de macrorrestos vegetales (Adesmia sp., Fabiana sp., y Parastrephia sp.), propias de pisos de altura, que fueron recuperados como parte de una actividad ritual (challa fundacional) en el sitio residencial Batungasta, a $1480 \mathrm{msnm}$ (Ratto et al., 2010, 2014, 2015). Estos casos constituyen ejemplos de la continuidad en el manejo de recursos vegetales silvestres y cultivados a lo largo del tiempo.

El análisis antracológico da cuenta de cambios y continuidades que coinciden con algunas tendencias observadas en el registro arqueológico y arqueobotánico regional. Quizás la más importante de las continuidades es la utilización de Prosopis sp. en todos los sectores de la región de Fiambalá, tendencia espacial que continúa en el tiempo desde las sociedades del primer milenio hasta el Estado incaico, lo cual no es extraño dado que el registro antracológico de Prosopis sp. se extiende desde el NOA hasta Patagonia (Andreoni, 2014; Ciampagna, 2015; Marconetto, 2008, entre otros). Además, el uso de la madera de Prosopis sp. tiene un amplio registro arqueológico en el NOA donde se lo ha identificado como material constructivo para postes o vigas en sitios emplazados en diferentes valles catamarqueños (Capparelli et al., 2003; Marconetto y Gordillo, 2008; Valencia y Balesta, 2013). De igual manera, está presente en ambiente altoandino tanto en sitios residenciales como de gran contenido simbólico (reservorio de leńa de Prosopis aff. alba; Ratto et al., 2010). Las fuentes bibliográficas mencionan el uso de Prosopis sp. para la confección de múltiples artefactos, como por ejemplo, tarabitas (Raviña et al., 2007), implementos del complejo alucinógeno, agrícolas (Sprovieri y Rivera, 2014) y para la elaboración de textiles, como es el caso de los torteros (Rodríguez, 2008). Una situación similar se aprecia en la utilización del algarrobo como materia prima para la preparación de alimentos y bebidas, lo que se evidencia a nivel de macro y microrrestos de este género en diversos contextos de Argentina (ver Capparelli et al., 2015; Giovannetti et al., 2008).

Análisis arqueobotánicos realizados en Puente del Diablo y Huachichocana III permitieron observar en ambos sitios un aumento en el consumo de Prosopis sp., fundamentalmente con el objeto de elaborar bebidas, añapa durante el Formativo y aloja durante el Tardío, preparación que se hace notable en el momento incaico en Huachichocana, en situaciones de congregación social (Lema et al., 2012). Asimismo, es interesante la asociación de macrorrestos ligados a estas bebidas en contextos funerarios. En Batungasta se identificaron artejos y semillas de Prosopis flexuosa, en contextos funerarios como Bebé de la Troya y restos de Prosopis afín P. nigra, P. Alexuosa o híbridos (Ratto et al., 2010, 2014). El uso de Prosopis sp. para la preparación de bebidas, posiblemente aloja, se evidencia en Batungasta por medio de la presencia de gránulos de almidón de este género en fragmentos cerámicos y en aríbalos del sitio San Francisco (puna de Chaschuil) y por medio de estudios de ácidos grasos (Lantos et al., 2015).

En los registros arqueológicos que aquí estudiamos se identificó Prosopis sp. en todos los sitios, excepto en un núcleo habitacional de la localidad de Palo Blanco (NH-03), siendo además el taxón mejor representado en los tres sectores analizados y a lo largo de toda la secuencia temporal. Evidentemente "el árbol", como se conoce al algarrobo, fue central en la organización social tanto de las sociedades del primer milenio como la tardía-incaica. La localización de los sitios Mishma-7 y Batungasta en medio de los algarrobales (los cuales en tiempo históricos cubrían gran parte del fondo del valle), el cultivo del taxón (sensu Palacios y Brizuela, 2005) y el manejo de los bosques demuestran su relevancia en la organización social de las poblaciones que habitaron el valle. La importancia del algarrobo ha sido señalada por Capparelli (2004 Ms.) al analizar el registro arqueobotánico del Shincal, cuando advierte la ausencia de carbones de Prosopis sp. y la elevada cantidad de carporrestos de este género, por lo cual considera que, al menos en el valle de Hualfin, los productos derivados del algarrobo debieron 
ser controlados por el Estado inca y que siguieron en importancia a la producción de maíz. Como se mencionó en la introducción, en otros sectores del área andina se ha propuesto el cultivo y manejo del algarrobo, como en Atacama (Agüero y Uribe, 2011; McRostie, 2014, 2016; Vidal, 2006) y en la costa sur de Perú (Bereford-Jones, 2011). Incluso se ha propuesto la domesticación del complejo Prosopis juliflora-P. pallida, no en sentido estricto, entendiendo como plantas domesticadas aquéllas que dependen del hombre para su reproducción, sino más bien, vinculado a múltiples técnicas de manejo de los recursos silvestres que incluyen prácticas silvícolas, las cuales pueden conllevar modificaciones fenotípicas tras sucesivas generaciones de selección y cultivo (Bereford-Jones, 2011). La presencia de algarrobales en zonas de cultivo en ambientes desérticos trae aparejada -como indica Bereford-Jones (2011)-, una serie de ventajas: aporta nitrógeno y aumenta la fertilidad de los suelos, disminuye los efectos de la erosión eólica y fluvial con un sistema radicular bien desarrollado. A esto podemos agregar un incremento en la producción de leña de buena calidad, materia prima para la construcción y confección de artefactos, además de ser un importante aporte en la dieta de los pueblos de la región, todo lo cual se evidencia en la región de Fiambalá desde tiempos formativos hasta la llegada del Estado incaico. Tampoco es un dato menor la consideración de los aspectos simbólicos y religiosos vinculados al algarrobo (Gentile, 2001; Karlovich, 2005).

Ahora bien, retomando el registro de Prosopis sp. en Fiambalá y considerando los Hornos de La Troya, en la periferia de Batungasta, se han registrado variaciones en su utilización, tanto vinculadas a sus formas como a la cronología (ver Tablas 1 y 2). De un total de siete hornos datados, solo se realizó el análisis antracológico en cinco, ya que en los dos restantes el material fue utilizado para dataciones. Si bien la muestra es reducida, se pueden observar algunas tendencias preliminares. En primer lugar, se observa una continuidad en el uso de Prosopis sp. e independientemente de la forma o la cronología de los hornos. Esto es coincidente con la identificación de semillas de Prosopis nigra y carbones de Prosopis sp., que fueron recuperados en otras dos estructuras tipo pera u ojo de cerradura de los Hornos de La Troya, excavadas por Caletti (2005 Ms.). Las varia- ciones se registran principalmente en los taxones acompañantes, ya que: a) en los hornos de forma circular (BATH-31/2, BATH-36 y BATH-35), Prosopis sp. se encuentra solo o combinado con Lithraea aff. molleoides; mientras que, b) los de forma de ojo de cerradura o pera (BATH-24/2) y BATH-03), de cronología posterior al primer milenio, presentan distintas combinaciones de Prosopis sp. asociado con Geoffroea decorticans, Acacia caven/Prosopis ferox, y muy baja frecuencia de Lithraea aff. molleoides, entre las cuales el algarrobo en todos los casos es la planta mejor representada.

En el caso del horno circular BATH-31/2, el cual presenta las paredes fuertemente termoalteradas, es posible que la combinación de Lithraea aff. molleoides y Prosopis sp. permitiera alcanzar mayores temperaturas que el uso exclusivo de Prosopis sp., ya que Lithraea aff. molleoides se utiliza en la fragua de metales (Capparelli y Raffino, 1997). Adicionalmente, creemos que la presencia de resinas facilita la producción de fuego, por lo tanto suponemos que la combinación de Lithraea aff. molleoides con Prosopis sp. debió permitir obtener temperaturas elevadas durante un mayor tiempo. Este aspecto tecnológico de la producción puede relacionarse con la mejor calidad de las piezas cerámicas del Período Formativo, especialmente por su mayor compacidad. En cuanto a la procedencia de Lithraea aff. molleoides, es difícil de establecer. Según Capparelli y Raffino (1997) esta especie crece en determinadas quebradas y forma pequeños bosques acompańados de Prosopis sp., por lo cual debió ser trasladado hasta el sitio desde algún otro sector del valle que actualmente desconocemos. Mientras que en momentos de la ocupación incaica se combinaron distintas maderas locales, la frecuencia de Lithraea aff. molleoides es muy baja en comparación con el período anterior, lo que puede interpretarse de múltiples maneras, que incluyen procesos culturales de reutilización de las estructuras, a cambios en la organización social, en el acceso a los recursos, en aspectos netamente tecnológicos e incluso de procesos de formación pre y post-depositacionales, entre otros. A diferencia de lo registrado en los Hornos de La Troya, en el sitio incaico del Shincal se han recuperado ramas y troncos Lithraea ternifolia y Bulnesia sp., pero en este caso se infiere que éstos formaron parte del techo de una estructura incendiada (Capparelli, 2004 Ms.). 
Si comparamos las secuencias antracológicas generadas en este trabajo y los antecedentes arqueobotánicos existentes (Ratto et al., 2010), observamos algunos cambios en los taxones utilizados para cada uno de los bloques temporales considerados. En este sentido, en la aldea formativa de Palo Blanco se registra una mayor diversidad de riqueza taxonómica, que incluye un amplio rango de maderas que pudieron ser colectadas en las inmediaciones del sitio o en otros sectores del valle. Entre éstas se encuentran maderas con diversas aplicaciones como pueden ser iniciadores de fuego, como por ejemplo, Acantholippia aff. salsoloides (Ratto et al., 2010), para la obtención de ceras (Cercidium praecox), junto con otras con diversas propiedades combustibles (Bulnesia aff. retama, Geoffroea decorticans, Rhamnaceae, Acacia sp., Schinus sp.), todas en contextos domésticos.

Por su parte, en el sitio incaico Batungasta existen claras diferencias entre los distintos contextos analizados, aunque la riqueza taxonómica es menor con respecto a la aldea formativa. Además, de las ya mencionadas en el caso de los hornos para la producción cerámica, Larrea sp., Schinus sp. y Rhamnaceae se registraron en contextos domésticos; mientras que Fabiana sp. y Parastrephia sp., en otros vinculados a prácticas rituales. Entre estos taxones, Parastrephia teretiuscula y Fabiana squamata, conocidas como koba entre otros nombres vernáculos, son utilizadas como incienso o para sahumar el ganado en los floreos realizados en el norte de Chile (Villagrán et al., 2003). En el NOA, las poblaciones de Antofalla (Catamarca) destinan la leña de Paratrephia lepidophylla para el uso doméstico y Parastrephia quadrangularis para el sahumado en rituales ceremoniales de veneración, agradecimiento y curación (Jofré, 2006). En Tebenquiche Chico la presencia de Parastrephia sp. en pozos excavados en una de las habitaciones, ha sido interpretada como una práctica de sahumado previo a la construcción de la vivienda (Jofré, 2006).

En la localidad de Mishma, la muestra analizada da cuenta de una baja riqueza taxonómica, donde Prosopis sp. fue el único taxón representado en el primer milenio (Formativo); mientras que Prosopis sp. y Geoffroea decorticans fueron identificados en momentos tardío-incaicos. Sobre el primer taxón ya hemos realizado extensas referencias, por lo que nos concentraremos en el segundo. Al respecto,
Geoffroea decorticans (chañar) es un árbol que proporciona madera dura a semidura que tiene múltiples aplicaciones en la elaboración y confección de objetos domésticos y para la construcción, además de ser un excelente combustible. También es utilizado con fines medicinales, para la elaboración de tinturas y como alimento (Delhey, 1991). El chañar presenta un amplio registro arqueológico; en este sentido se ha identificado como material constructivo en distintos sitios de Catamarca (Marconetto y Gordillo, 2008; Valencia y Balesta, 2013); como materia prima para la confección de tarabitas (Ravińa et al., 2007); como leńa en distintos sitios del valle de Ambato (Marconetto, 2008) y en diferentes sectores de la región centro-oeste (Andreoni, 2014); y como importante fuente de alimento según lo demuestra el registro arqueológico de carporrestos en Ambato (Marconetto et al., 2009). En la región de Chaschuil se han identificado semillas de Geoffroea decorticans en asociación a un fogón del Período Formativo (650-750 DC) del sitio Fiambalá 1 (5000 msnm) y en contextos funerarios tardíos del sitio Bebé de la Troya (Ratto et al., 2010, 2014).

En síntesis, los estudios antracológicos presentados evidencian una continuidad en las prácticas de selección y utilización de leña, donde las diferencias observadas están dadas, por una parte, por los contextos del cual proceden (domésticos y productivos) $y$, por otra, por la oferta ambiental de recursos combustibles de cada uno de los sectores de la región de Fiambalá. Sin embargo, se han evidenciado cambios a lo largo del tiempo en los que se destaca la concentración en el uso de Prosopis sp. y la disminución en la riqueza taxonómica, en contextos incaicos, posiblemente vinculados a cambios en la organización social y en los mecanismos de apropiación que conllevó la conquista. Un aspecto relevante que se desprende de los estudios antracológicos y de los carporrestos vinculados a la selección/utilización de Prosopis sp. con múltiples usos, es la existencia de algarrobales cultivados en las proximidades del sitio Batungasta (actuales pueblos de El Puesto y Anillaco, Departamento Tinogasta, Catamarca). Con ello, podemos afirmar que el algarrobo fue central en la subsistencia de las poblaciones, tanto del primer milenio como del Período Tardío-Inca. En cuanto al manejo de los bosques implantados, creemos que durante el establecimiento del Estado inca 
en la región de Fiambalá el control de éstos pasó a sus manos. Sin embargo, es probable que las técnicas de manejo no fueran desconocidas por las sociedades formativas, dado que para este momento se han registrado procesos similares en otros sectores de la región andina (p.e., en San Pedro de Atacama y sur de Perú). La apropiación de los algarrobales llevada a cabo por el incario fue posible debido a que las poblaciones locales ya poseían conocimientos sobre el manejo de los bosques. Entre ellos puede incluirse la tolerancia de ciertos ejemplares en campos de cultivo, la eliminación sistemática de competidores, o prácticas silvícolas como la poda y el riego. Esto permitió el establecimiento de nuevas prácticas y la perpetuación de otras que pudieron haber sido desarrolladas desde tiempos previos a la llegada de los incas.

\section{Agradecimientos}

La investigación se realizó en el marco de una Beca Superior del primer autor en el marco del proyecto PICT-2012-0196 dirigido por la Dra. N. Ratto, en la cual se integraron estudios previos realizados bajo la supervisión de la Dra. B. Marconetto. Agradecemos especialmente a la Dra. Aylen Capparelli por la disponibilidad de los equipamientos y colecciones del Laboratorio 129 de la División de Arqueología del Museo de La Plata Facultad de Ciencias Naturales y Museo y al Lic. Luis Coll por realizar la Figura 1.

\section{Referencias citadas}

Agüero, C. y Uribe, M. (2011). Las sociedades Formativas de San Pedro de Atacama: Asentamiento, cronología y proceso. Estudios Atacameños, Arqueología y Antropología Surandinas, 42, 53-78.

Andreoni, D. (2014). Plantas leñosas y estrategias humanas en el sur de Mendoza: una aproximación arqueobotánica. Tesis doctoral. Facultad de Ciencias Naturales y Museo, Universidad Nacional de La Plata, La Plata.

Bereford-Jones, D. (2011). The lost woodlands of Ancient Nasca. A case-study in ecological and cultural collapse. British Academy Postdoctoral Monograph Series. Oxford: Oxford University Press.
Bereford-jones, D., Whaley, O., Ledesma, A. C. y Cadwallader, A. (2011). Two millennia of changes in human ecology: archaeobotanical and invertebrate records from the lower Ica valley, south coast Peru. Vegetation History and Archaeobotany, 20(4), 273-292.

Bonomo, N., Osella, A. y Ratto, N. (2010). Detecting and mapping buried buildings with GPR at an ancient village in Northwestern Argentina. Journal of Archaeological Science, 37(12), 3247-3255.

Burkart, A. (1976). A monograph of the genus Prosopis (Leguminosae subfam. Mimosoideae). (Parts 1 and 2). Catalogue of the recognized species of Prosopis. Journal of the Arnold Arboretum, 57(3), 219-249 y 57(4), 450-525.

Burkart, A. y Simpson, B. B. (1977). The genus Prosopis and annoted key to the species of the world. En B. B. Simpson (Ed.). Mesquite: its biology in two desert ecosystems (pp. 201-215). Stroudsburg, Pennsylvania: Dowden, Hutchinson and Ross.

Cabrera, A. (1976). Regiones fitogeográficas argentinas. Enciclopedia Argentina de Agricultura y Jardinería. Tomo II(1), 347-411.

Caletti, S. (2005) Ms. Tecnología de cocción de piezas de arcilla. Un caso de estudio: Batungasta, Dpto. Tinogasta, Catamarca, Argentina. Tesis de Licenciatura. Escuela de Arqueología, Universidad Nacional de Catamarca, Catamarca.

Capparelli, A. (2004) Ms. History of plant-people relationship and deforestation at the Hualfin Valley (Argentina), since Inca occupation (1471 AD) to the present day. Final report International Foundation for Science, grant number D-3118-1, Stockholm.

Capparelli, A. y Raffino, R. (1997). La etnobotánica de "El Shincal" (Catamarca) y su importancia para la arqueología I: recursos combustibles y madereros. Parodiana, 10(1-2), 181-188.

Capparelli, A., Zagorodny, N. y Balesta, B. (2003). Wood remains from Andean Argentina: the use of Prosopis sp. L. in hut construction. Journal of Etnobiology, 23(1), 143-154. 
Capparelli, A., Pochettino, M. L., Lema, V., López, M. L., Andreoni, D., Ciampagna, M. L. y Llano, C. (2015). The contribution of ethnobotany and experimental archaeology to interpretation of ancient food processing: methodological proposals based on the discussion of several case studies on Prosopis spp., Chenopodium spp. and Cucurbita spp. from Argentina. Vegetation History and Archaeobotany, 24(1), 151-163.

Ciampagna, M. L. (2015). Estudio de la interacción entre grupos cazadores recolectores de Patagonia y las plantas silvestres: el caso de la costa norte de Santa Cruz durante el Holoceno Medio y Tardío. Facultad de Ciencias Naturales y Museo, Universidad Nacional de La Plata, Buenos Aires.

Czajka, W. y Vervoorst, F. (1956). Die naturräumlinche Gliederung Nordwest-Argentinieens. Petermanns Geographischen Mitteilungen. 100( 2),89-109, y 100(3), 196-208.

Delhey, R. (1991). El chañar (Geoffroea decorticans, Leguminosae). Etnobotánica y utilización. Parodiana, 6(2), 337-362.

Feely, A. (2010). Estilos tecnológicos y tradiciones de manufactura cerámica durante momentos formativos en el oeste de Tinogasta (Catamarca). Arqueología Argentina en el Bicentenario de la Revolución de Mayo. XVII Congreso Nacional de Arqueología Argentina, Tomo I (pp. 199-204). Facultad de Filosofía y Letras, Universidad Nacional de Cuyo, Mendoza.

Feely, A. (2011). Caracterización de estructuras de doble cámara para la cocción de artefactos cerámicos en La Troya (Tinogasta, Catamarca). Relaciones de la Sociedad Argentina de Antropología, XXXVI, 325-330.

Feely, A. y Ratto, N. (2009). Variaciones de los conjuntos cerámicos de unidades domésticas: aldeas y puestos formativos del bolsón de Fiambalá (ca. 1500-1300 AP). En N. Ratto (Comp.). Entrelazando ciencias: sociedad y ambiente antes de la conquista española (pp. 99-129). Buenos Aires: Eudeba.

Gentile, M. (2001). Chiqui: etnohistoria de una creencia andina en el Noroeste Argentino. Bulletin de l'Institut Français d'Études Andines, 30(1), 27-102.

Giovannetti, M. A., Lema, V. Bartoli, C. G. y Capparelli, A. (2008). Starch grain characterization of Prosopis chi- lensis (Mol.) Stuntz and P. flexuosa DC, and the analysis of their archaeological remains in Andean South America. Journal of Archaeological Science, 35(11), 2973-2985.

Jofré, C. (2006). Arqueología del fuego. Un estudio de las prácticas domésticas asociadas al fuego en Tebenquiche Chico. Tesis de Licenciatura. Universidad Nacional de Catamarca, Catamarca.

Karlovich, A. (2005). El canto del chiqui: texto y contexto. Guaca, 1(2), 21-32.

Lantos, I., Spangenberg, J. E., Giovannetti, M. A., Ratto, N. y Maier, M. S. (2015). Maize consumption in preHispanic south-central Andes: microscopic and chemical evidence from organic residues in pottery from west Tinogasta (Catamarca, Argentina). Journal of Anchaeological Science, 55(1), 83-99.

Lema, V., Capparelli, A. y Martínez, A. (2012). Las vías del algarrobo: antiguas preparaciones culinarias en el Noroeste Argentino. En Babot, P., Marschoff, M. y Pazzarelli, F. (Eds.). Las manos en la masa. Arqueologías, antropologías e historias de la alimentación en Suramérica (pp. 639-666). Córdoba: ISES/IDACOR.

Marconetto, M. B. (2008). Recursos forestales y el proceso de diferenciación social en tiempos prehispánicos. Valle de Ambato, Catamarca. BAR South American Archaeology Series 3, Oxford.

Marconetto, M. B. y Gordillo, E. I. (2008). "Los techos del vecino": análisis antracológico de restos de construcción carbonizados de los sitios "Iglesia de los Indios" y "Piedras Blancas" (Catamarca). Darwiniana, 46(2), 213-226.

Marconetto, M. B., Dantas, M., Gastaldi, M., Figueroa, G., Martínez, G., Lindskoug, H., Pazzarelli, F. y Laguens, A. (2009). Mil chañares. Prácticas asociadas a Geoffroea decorticans en Aguada de Ambato a fines del primer milenio. En Libro de resúmenes del V Congreso Internacional de Etnobotánica (pp. 42). San Carlos de Bariloche: Fusiongráfica La Plata.

Martino, L., Bonomo, N., Lascano, E., Osella, A. y Ratto, N. (2006). Geoelectrical and GPR joint prospection in the ancient Palo Blanco archaeological site, NW Argentina. Geophysics, 71(6), 193-199. 
McRostie, V. (2014). Arboricultura y silvopastoralismo en el Período Formativo (1400 a.C.-500 d.C.) de la cuenca del Salar de Atacama. Chungara. Revista de Antropología Chilena, 46(4), 543-557.

McRostie, V. (2016). Algarrobales de Atacama. ¿Nativos o exóticos? ¿Silvestres o domésticos? Actas del XIX Congreso Nacional de Arqueología Argentina. Serie Monográfica y Didáctica 58 (pp. 1539-1544). Tucumán: Facultad de Ciencias Naturales e Instituto Miguel Lillo de la Universidad Nacional de Tucumán.

Miyano, J. P., De Nigris, M. y Ratto, N. (2015). Zooarqueología de la Aldea de Palo Blanco (Tinogasta, Catamarca). Revista del Museo de Antropología, 8(2), 7-20.

Morello, J. (1958). La Provincia fitogeográfica de Monte. Opera Llilloana, II, 7-151.

Orgaz, M., Feely, A. y Ratto, N. (2007). La cerámica como expresión de los aspectos socio-políticos, económicos y rituales de la ocupación Inka en la puna de Chaschuil y el valle de Fiambalá (Dpto. Tinogasta, Catamarca). En Nielsen, A., Rivolta, M. C., Seldes, V., Vázquez, M. y Mercolli, P. (Comps.). Procesos sociales prehispánicos en el sur andino. La vivienda, la comunidad y el territorio (pp. 239-250). Córdoba: Brujas.

Orgaz, M. y Ratto, N. (2015). Estrategias de ocupación incaica en el sur del Noroeste Argentino (Departamento Tinogasta, Catamarca): la apropiación de paisajes sagrados y la memoria social. Nawpa Pacha. Journal of Andean Archaeology, 35(2), 217-23.

Palacios, R. y Brizuela, M. (2005). Prosopis: historia y elementos para su domesticación. Agrociencia, IX(1-2), 41-51.

Pasiecznik, N., Felker, P., Harris, P., Harsh, L., Cruz, G., Tewari, J., Cadoret, K. y Maldonado, L. (2001). The Prosopis juliflora complex: a monograph. Coventry, UK: Henry Doubleday Research Association HDRA. ISBN: 0-905343-30-1.

Pérez, M. C. y Ratto, N. (2004). Las plantas silvestres como recursos en las regiones áridas (Puna y Patagonia). En Crivalero, M. T., P. Fernández, P. M., y Guráieb, A. M. (Comps.). Contra viento y marea. Arqueología de Patagonia (pp. 295-310). Buenos Aires: Instituto Nacional de Antropología y Pensamiento Latinoamericano, Sociedad Argentina de Antropología.
Pique í Huerta, R. (1999). Producción y uso de combustible vegetal arqueológico: una evaluación arqueológica. Treballs d'Etnoarqueología 3. Madrid: Universidad Autónoma de Barcelona.

Ratto, N. (2005). La arqueología del Bolsón de Fiambalá a través de los Estudios de Impacto (Dpto. Tinogasta, Catamarca, Argentina). Actas dos I Jornadas Internacionais Vestígios do Passado. Lisboa: AGIR. Associação para a Investigação e Desenvolvimento Sócio-cultural.

Ratto, N. (2007). Paisajes arqueológicos en el tiempo: la interrelación de ciencias sociales, físico-químicas y paleoambientales (Dpto. Tinogasta, Catamarca, Argentina). En Producción y circulación prehispánicas de bienes en el sur andino, Nielsen, A., Rivolta, M.C., Seldes, V., Vázquez, M., y Mercolli, P. (Comps.). Colección Historia Social Precolombina, vol. 2 (pp. 35-54). Córdoba: Editorial Brujas.

Ratto, N. (Comp.) (2013). Delineando prácticas de la gente del pasado: los procesos socio-históricos del oeste catamarqueño (p. 502.) Buenos Aires: Sociedad Argentina de Antropología.

Ratto, N. (2015). Vivencias, acciones y resultados del quehacer arqueológico en el oeste de Tinogasta (Catamarca, Argentina). Corpus [en línea], 5(2). Publicado 17.12.15. URL: http:// corpusarchivos.revues.org/1511

Ratto, N. y Basile, M. (2010). La localidad arqueológica de Palo Blanco: nuevas evidencias. Arqueología Argentina en el Bicentenario de la Revolución de Mayo. XVII Congreso Nacional de Arqueología Argentina. Tomo IV (pp. 17071712). Mendoza: Facultad de Filosofía y Letra Universidad Nacional de Cuyo.

Ratto, N. y Boixadós, R. (2012). Arqueología y etnohistoria. La construcción de un problema de investigación (Abaucán, Tinogasta, Catamarca). Memoria Americana, 20(2), 187-220.

Ratto, N., Orgaz, M. y Plá, R. (2002). Producción y distribución de bienes cerámicos durante la ocupación inca entre la región puneña de Chaschuil y el valle de Abaucán (Dpto. Tinogasta, Catamarca). Relaciones de la Sociedad Argentina de Antropología, XXVII, 271-301. 
Ratto, N., Orgaz, M. y Plá, R. (2004). La explotación del Alfar de La Troya en el tiempo: casualidad o memoria (Departamento Tinogasta, Catamarca, Argentina). Chungara. Revista de Antropología Chilena, 36(2), 349-361.

Ratto, N., Rodríguez, M. F. y Hershey, D. (2010). Explotación y uso de recursos vegetales en sitios arqueológicos del área cordillerana y del valle mesotérmico (Depto. Tinogasta, Catamarca, Argentina). Actas del XVII Congreso Nacional de Arqueología Chilena. Tomo II (pp. 12671278). Valdivia.

Ratto, N., Montero, C. y Hongn, F. (2013). Environmental instability in western Tinogasta (Catamarca) during Middle Holocene and its relation to regional cultural development. Quaternary International, 307, 58-65.

Ratto, N., Bonomo, N. y Osella, A. (2013). Arqueo-geofísica en instalaciones del área del abanico aluvial del río La Troya (Departamento Tinogasta, Catamarca). En Ratto, N. (Comp.). Delineando prácticas de la gente del pasado: los procesos socio-históricos del oeste catamarqueño (pp. 337354). Buenos Aires: Sociedad Argentina de Antropología.

Ratto, N., Lema, V. y López, M. L. (2014). Entierros y ofrendas: prácticas mortuorias, agrícolas y culinarias en los siglos XIII y XIV en Tinogasta (Catamarca, Argentina). Darwiniana, 2(1), 125-143.

Ratto, N., Basile, M., Feely, A., Lantos, I., Coll, L., Carniglia, D. y Miyano, J. P. (2015). La gente y sus prácticas en las tierras bajas y altas del oeste tinogasteño en los siglos I al XIII (Catamarca, Argentina). En Korstanje, A., Lazzari, M., Basile, M., Bugliani, M. F., Lema, V., Pereyra Domingorena, L., y Quesada, M. (Eds.). Crónicas materiales precolombinas. Arqueología de los primeros poblados del Noroeste Argentino (pp. 215-245). Buenos Aires: Sociedad Argentina de Antropología.

Raviña, M., Fernández, A. M. y Capparelli, A. (2007). La relación de las tarabitas, horquetas o ganchos de atalaje con el tráfico de bienes en momentos tardíos prehispánicos. Estudios Atacameños, Arqueología y Antropología Surandinas, 33, 87-104.

Rodríguez, M. F. (2008). Recursos vegetales y tecnofacturas en un sitio arqueológico de la Puna Meridional argentina, Área Centro-Sur andina. Darwiniana, 46, 240-257.
Rojas, F. (2013). Rol de la minería y el ferrocarril en el desmonte del oeste riojano y catamarqueño (Argentina) en el período 1851-1942. Población y Sociedad, 20(2), 99-123.

Salinas, R. S. (2006). Ms. Informe flora y vegetación campo Giusepe 4 Anillaco, Dpto. Tinogasta, Catamarca. Manuscrito en poder del autor.

Sempé, M. C. (1976). Ms. Contribución a la arqueología del valle de Abaucán. Tesis doctoral. Facultad de Ciencias Naturales y Museo, Universidad Nacional de La Plata, La Plata.

Sempé, M. C. (1983). Mishma No7. Sitio incaico del valle de Abaucán. Dpto. Tinogasta, Pcia. Catamarca. Revista del Museo de La Plata. Antropología N.S. VIII(65), 405-438.

Sprovieri, M. y Rivera, S. M. (2014). Las maderas de la "Colección La Paya". Circulación y consumo en el valle Calchaquí (Salta). Intersecciones en Antropología, 15(1), 89-102.

Valencia, M. C. y Balesta, B. (2013). ¿Abandono planificado? Restos forestales carbonizados en sitios arqueológicos de La Ciénaga (Catamarca, Argentina). Bulletin de l'Institut Français d'Études Andines, 42(2), 145-172.

Vervoorst, F. (1951). Resultados de un viaje a la cuenca de Laguna Verde (Tinogasta, Catamarca). Actas de la XV Semana de Geografía (pp. 61-67). Facultad de Filosofía y Letras, Universidad Nacional de Cuyo, Instituto de Historia y Disciplinas Auxiliares, Sociedad Argentina de Estudios Geográficos, Buenos Aires.

Vidal, A. (2006). Patrones de uso de los recursos vegetales durante el período Formativo (1000 AC-500 DC) en los oasis de San Pedro de Atacama. Actas del XVII Congreso Nacional de Arqueologia Chilena, vol. 2 (pp. 1291-1302). Valdivia.

Villagrán, C., Romo, M. y Castro, V. (2003). Etnobotánica del sur de los Andes de la primera región de Chile: un enlace entre las culturas altiplánicas y las de quebradas altas del Loa Superior. Chungara. Revista de Antropología Chilena 35(1), 73-124. 